\title{
The time course of intentional binding
}

\author{
Miriam Ruess $^{1} \cdot$ Roland Thomaschke $^{1} \cdot$ Andrea Kiesel $^{1}$
}

Published online: 9 February 2017

(C) The Psychonomic Society, Inc. 2017

\begin{abstract}
Stimuli caused by actions (i.e., effects) are perceived earlier than stimuli not caused by actions. This phenomenon is termed intentional binding (IB) and serves as implicit measure of sense of agency. We investigated the influence of effect delay and temporal predictability on IB, operationalized as the bias to perceive the effect as temporally shifted toward the action. For short delays, IB increased with delay (Experiment 1: $200 \mathrm{~ms}, 250 \mathrm{~ms}, 300 \mathrm{~ms}$ ). The initial increase declined for longer delays (Experiment 2: $100 \mathrm{~ms}$, $250 \mathrm{~ms}, 400 \mathrm{~ms}$ ). This extends previous findings showing IB to decrease with increasing delays for delay ranges of $250 \mathrm{~ms}$ to $650 \mathrm{~ms}$. Further, the hypothesis that IB, that is, sense of agency, might be maximal for different delays depending on the specific characteristics and context of action and effect, has important implications for human-machine interfaces.
\end{abstract}

Keywords Temporal predictability · Action-effect interval · Temporal binding $\cdot$ Intentional binding $\cdot$ Effect delay $\cdot$ Sense of agency

We manipulate our environment according to our goals. More precisely, we act to produce intended effects. Interestingly, we fail to perceive our intended effects in a temporally correct manner. Effects are temporally shifted toward the actions that produced them (Haggard, 2005). This temporal bias does not occur for effects of passive actions (Nolden, Haering, \& Kiesel, 2012), actions triggered by transcranial magnetic stimulation (TMS;

Miriam Ruess

ruess@psychologie.uni-freiburg.de

1 Cognition, Action, and Sustainability Unit, Department of Psychology, Albert-Ludwigs-University of Freiburg, Germany 79085 Freiburg
Haggard, Clark, \& Kalogeras, 2002), inhibited actions (Haggard, Poonian, \& Walsh, 2009), or actions that are caused by rubber hands (Engbert, Wohlschläger, \& Haggard, 2008). Thus, the temporal bias seems to be restricted to effects caused by intentional actions, like executed or observed key presses at freely chosen points in time (Poonian \& Cunnington, 2013). Consequently, the phenomenon is termed intentional binding (IB), or temporal binding, and it serves as an important implicit measure of sense of agency (Moore, Wegner, \& Haggard, 2009).

Sense of agency refers to the feeling that a certain external event was caused by oneself. Such a feeling can come in different degrees of certainty or confidence, which varies systematically with the magnitude of IB (Moore \& Haggard, 2010). Sense of agency is an increasingly important measure in assessing the feeling of control in current applied interface ergonomics - for example, in aviation or concerning input modalities to prosthetic arms (e.g., Berberian, Sarrazin, LeBlaye, \& Haggard, 2012; Limerick, Coyle, \& Moore, 2014).

The classic method to measure sense of agency in terms of $\mathrm{IB}$, which we also employed in our experiments, asks for estimates of points in time and mostly applies a classic clock procedure (Haggard et al., 2002; Libet, Gleason, Wright, \& Pearl, 1983; Wundt, 1887): Participants see a rotating clock hand while they execute an action followed by a tone. Afterwards, they are asked to estimate the position of the clock hand at tone occurrence. These estimates are compared to time-point estimates in a baseline condition where the tone occurred without a preceding action. Usually, the point in time of the tone is estimated to occur earlier if the tone is caused by the executed action in comparison to when it occurred without preceding action. The magnitude of IB is commonly computed as the difference between these conditions, as it reflects the amount of the temporal "shift" of the perceived effect toward the action.

An important issue is whether IB, respectively sense of agency, depends on the delay of the effect. That is, when the 
interval between action and effect gets longer, does the perceived effect shift become stronger or weaker? A number of studies addressed a similar question by assessing IB in the sense of a shortening of the perceived duration of actioneffect intervals. Those studies observed increased IB with increasing delays (e.g., Humphreys \& Buehner, 2009; Nolden et al., 2012; Wen, Yamashita, \& Asama, 2015).

However, to our knowledge, only one study addressed the question as to how the delay of the effect influences the perceived point in time of the effect. Haggard et al. (2002) observed decreased IB with increasing delays when varying delays in the range of $250 \mathrm{~ms}$ to $650 \mathrm{~ms}$. This means that the more time had elapsed before the effect followed the action, the less IB was found. Furthermore, temporal predictability was manipulated by presenting the effect either always after the same, temporally predictable delay in one condition, or after a variable, temporally unpredictable delay in another condition. IB was reduced for temporally unpredictable in comparison to temporally predictable effects, and this temporal predictability interacted with delay duration. Numerically, IB decreased faster for temporally predictable in comparison to unpredictable effects, though a statistical evaluation of this tendency was not reported.

The implications of the study by Haggard et al. (2002) for everyday life actions are rather limited because three delays with a relatively large range were used $(250 \mathrm{~ms}, 450 \mathrm{~ms}$, and $650 \mathrm{~ms}$ ), whereas in everyday life we commonly deal with somewhat shorter delays: For example, in human-machine interactions, effects typically occur about $250 \mathrm{~ms}$ after action execution (Seow, 2008; Smith \& Mosier, 1986). Furthermore, the range of temporal deviations in most of our interaction environments is typically smaller than $200 \mathrm{~ms}$. Thus, an open question is how IB varies for shorter effect delays with small delay variations, that is, in settings that are more closely related to our everyday life interactions.

Furthermore, answers to this question might offer insights on how, for example, machines like a coffee machine or airplane cockpit should be programed to ensure maximal perceived control over the effects, that is, maximal sense of agency (Berberian et al., 2012). In this context, it is particularly important to further investigate how delay influences IB, that is, sense of agency, especially if effect delays vary around the (for human-machine interactions typical) delay of $250 \mathrm{~ms}$ (Seow, 2008; Smith \& Mosier, 1986). To this end, we assessed IB with a small range (200 ms, $250 \mathrm{~ms}, 300 \mathrm{~ms}$; Experiment 1) and an extended range (100 ms, $250 \mathrm{~ms}, 400 \mathrm{~ms}$; Experiment 2) around the delay of $250 \mathrm{~ms}$, whereby effect delays varied either predictably or unpredictably, like in the study by Haggard et al. (2002).

This also entails, however, the application of an extremely short delay $-100 \mathrm{~ms}$. The dependency of IB on delays shorter than those applied by Haggard et al. (2002) is unclear. Their results suggest a monotonously decreasing relationship of effect delay and IB. This means IB would get stronger with decreasing delay duration. Such a finding in our study would be especially interesting because we employ very short effect delays. Particularly strong IB for short delays might lead to a violation of the order principle of causality (Hume, 1874). This principle states that causes must precede their consequences. Assuming that the temporal perception of the action remains rather unbiased, a very strong IB for short delays might lead to such a strong shift of the perceived point in time of the effect that it might be perceived even before its cause, the action. This is why it is especially important to investigate how IB is affected by short effect delays.

Note that IB is also characterized by a perceived temporal shift of the action toward the effect (Haggard et al., 2002). To our knowledge, however, no previous study addressed the question of whether the shift of the action (action IB) is influenced by effect delay. Further, as far as we know, only one previous study manipulated temporal predictability and measured IB as shift of the action (Stenner et al., 2014). In this study, action IB was analyzed separately for three different randomly presented delays. Yet these analyses are difficult to interpret because the shift was measured in trials where the action caused an effect (experimental trials), which were not blocked but randomly intermixed with trials where the action did not cause any effect. Thus, in these experimental trials participants did not actually expect their action to be followed by an effect, which also might have affected action IB to some degree. Consequently, it provides no direct implications concerning how action IB might be influenced by effect delay, or by temporal predictability. Nevertheless, we measured IB also for the action and provide an exploratory analysis of its dependence on delay.

\section{Experiment 1}

To assess IB, participants were asked to press one of two possible response keys at a freely chosen point in time. In the experimental conditions, each key press contingently produced one of two possible effect tones (Desantis, Hughes, \& Waszak, 2012). The tone occurred after a delay of $200 \mathrm{ms,}$ $250 \mathrm{~ms}$, or $300 \mathrm{~ms}$. The delay varied either trial-wise or blockwise, so that the delay of an effect was either unpredictable (varying trial-wise) or predictable (varying block-wise). In some blocks, participants were asked to judge the position of a rotating clock hand at the moment when they pressed the response key (action experimental). In other blocks, they had to judge the hand's position when they heard the effect tone (effect experimental). In baseline conditions, just an action was required, without a following tone (action baseline), or the tone occurred without preceding action (effect baseline), and participants were also asked to estimate the respective points in time in relation to the rotating clock hand. IB was measured as the difference of experimental conditions of 
action and effect compared to respective baseline conditions of action and effect (cf. Haggard et al., 2002).

\section{Method}

Participants Based on effect sizes in previous studies (e.g., Haering \& Kiesel, 2014), we tested 48 participants (34 females; mean age $=26$ years, $S D=9.15$, range: $18-61$ years; 47 right-handed), in exchange for 15 euros or course credit.

Apparatus and stimuli The experiment was run using the EPrime 2.0 software (Schneider, Eschmann, \& Zuccolotto, 2012) on a standard PC with a 20 -in. LCD screen $(1,600$ pixels $\times 1,200$ pixels, $60 \mathrm{~Hz}$ refresh rate). Two separate external response keys were operated with the index and middle finger of the left hand. Two sine tones $(400 \mathrm{~Hz}$ or $800 \mathrm{~Hz})$ were presented as effects by Auna Base DJ 10014216 headphones for $150 \mathrm{~ms}$. They were mapped to response keys in a SMARC-compatible manner (Mudd, 1963), that is, left key to $400 \mathrm{~Hz}$ and right key to $800 \mathrm{~Hz}$. Participants watched a centrally presented clock face (diameter $6.3 \mathrm{~cm}$, clock hand $2.2 \mathrm{~cm}, 2,560 \mathrm{~ms} /$ full rotation) with 12 labeled "minute" intervals (see Fig. 1). Time estimates were given with the right hand using the number pad of the keyboard (1-9).

Procedure The experiment consisted of two 1-hour sessions conducted on two different days: In one session, the three effect delays varied between blocks (temporally predictable effects), whereas they varied within blocks in the other session (temporally unpredictable effects). The order of sessions was counterbalanced across participants.
We employed the so-called classic clock procedure (Haggard et al., 2002) with the Libet Clock (Libet et al., 1983; Wundt, 1887) that involves the visual display of an analogue clock, that is, a hand revolving over a dial at a continuous pace. We used this clock as a reference for the participants' time estimates (see Fig. 1): Each trial started with the presentation of the clock on the screen, and the clock hand immediately started to rotate at a random position. Participants were instructed to press one of the two keys at a freely chosen point in time, but to wait until the clock hand had revolved at least once, and not to press at a preplanned point in time or clock position. Further, they were instructed to randomly choose which key to press, and to press each key approximately equally. In the experimental conditions, the action was followed by the effect, a tone, with $200 \mathrm{~ms}, 250 \mathrm{~ms}$, or $300 \mathrm{~ms}$ delay. In the baseline conditions, no tone followed after action execution (action baseline) or no action was required, and one of the two tones was presented randomly $2,560 \mathrm{~ms}$ to $5,120 \mathrm{~ms}$ after the trial started (effect baseline). The clock disappeared in all conditions at a random time, $2 \mathrm{~s}$ to $3 \mathrm{~s}$ after the tone (or after action execution in the action baseline condition). In the experimental and baseline condition for the action, participants were asked to estimate the position of the clock hand at the moment they pressed the key, whereas in the experimental and baseline condition for the effect, they were prompted to estimate the position of the clock hand at tone onset (in minutes 1-60).

Each of the two sessions started with one baseline block for the action and one baseline block for the effect, followed by six experimental blocks in which action and effect judgments alternated block-wise, and finished with another baseline block for action and effect, respectively. The order of whether

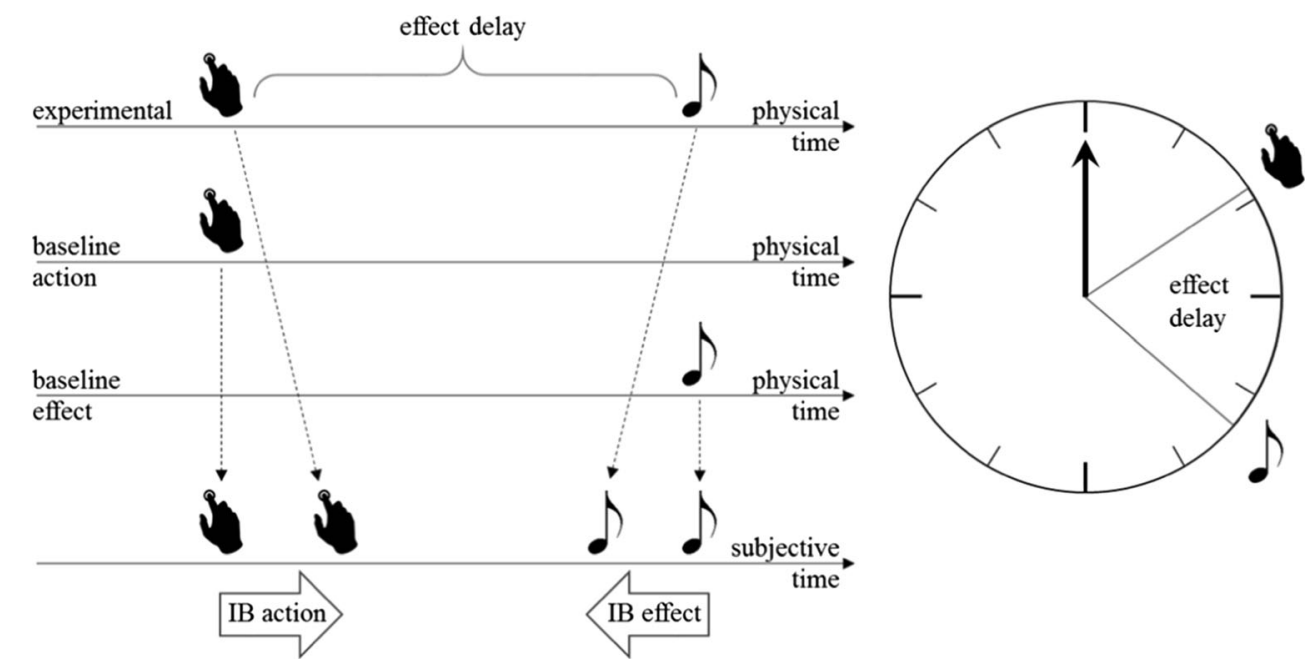

Fig. 1 Employed clock procedure (Haggard et al., 2002). Participants saw a rotating clock hand while asked to press a key that was followed by a tone (experimental conditions). In the baseline condition, the key press was not followed by the tone (baseline action) or the tone was presented without preceding action (baseline effect). After the clock hand stopped, participants had to judge the position of the clock hand at action execution (experimental and baseline condition action) or tone onset (experimental and baseline condition effect). Intentional binding was calculated as the difference between the mean judgments in experimental and baseline conditions for action and effect separately 
action or effect judgements were required first was counterbalanced across participants. Each of the baseline blocks consisted of 21 trials ( 21 trials $\times 2$ blocks $=42$ trials $)$, and the experimental blocks consisted of 42 trials (unpredictable session: 14 trials per delay $\times 3$ blocks $=42$ trials per delay overall per session). The three delays were presented randomly in the experimental blocks of the unpredictable session. In the experimental blocks of the predictable session, the same delay was presented in two consecutive blocks and the order of the delays was counterbalanced across participants. Both sessions started with three training trials for each baseline and experimental condition, respectively.

For each participant, the difference between estimated and actual positions of the clock hand for either action or effect was computed trial-wise and averaged separately for each condition (baseline vs. experimental condition, action vs. effect condition, predictable vs. unpredictable session, delay $200 \mathrm{~ms}, 250 \mathrm{~ms}$, or $300 \mathrm{~ms}$ ). The angle differences were transformed into temporal differences (angle difference $\times$ $2,560 \mathrm{~ms} / 60$ ). If the trial-wise differences deviated more than $+/-2.5$ standard deviations from the participant's mean estimate in the respective condition, they were discarded (on average, $2.27 \%$ for each participant; for a similar procedure, see Haering \& Kiesel, 2014). Finally, IB was calculated as the difference between the mean shift of the perceived time in the baseline and experimental condition (separately for action vs. effect condition, predictable vs. unpredictable session, delay $200 \mathrm{~ms}, 250 \mathrm{~ms}$, or $300 \mathrm{~ms}$ ). We computed the differences in the way that both measures became positive when IB occurred: Therefore, results are reported as experimental minus baseline condition for action judgments and as baseline minus experimental condition for effect judgments. Consequently, positive values for IB action mean that the action is perceived later, that is, shifted toward the effect, and positive values for IB effect mean that the effect is perceived earlier, that is, shifted toward the action.

\section{Results}

IB for effects To assess whether IB was significant for each condition, we conducted separate $t$ - tests. All conditions showed significant IB, predictable, $200 \mathrm{~ms}: t(47)=7.12, M$ $=87.85, S E=12.34, p<.001 ; 250 \mathrm{~ms}: t(47)=7.90, M=$ 107.36, $S E=13.59, p<.001 ; 300 \mathrm{~ms}: t(47)=7.26, M=$ 120.53, $S E=16.60, p<.001$; unpredictable, $200 \mathrm{~ms}: t(47)=$ $5.25, M=71.86, S E=13.68, p<.001 ; 250 \mathrm{~ms}: t(47)=6.66, M$ $=98.24, S E=14.75, p<.001 ; 300 \mathrm{~ms}: t(47)=7.60, M=$ $124.79, S E=16.42, p<.001$.

A within-subjects $3 \times 2$ ANOVA (delay and predictability) revealed that IB increased for longer delays, $F(2,94)=26.08, p$ $<.001, \eta_{\mathrm{p}}{ }^{2}=.36 ; 200 \mathrm{~ms}: M=79.85, S E=12.28 ; 250 \mathrm{~ms}: M=$ $102.80, S E=13.56 ; 300 \mathrm{~ms}: M=122.66, S E=15.77$. Neither predictability, $F(1,47)=0.85, p>.250, \eta_{\mathrm{p}}{ }^{2}=.02$; predictable: $M=105.24, S E=13.25$; unpredictable: $M=98.30, S E=$ 14.77 , nor the interaction delay $\times$ predictability were significant, $F(2,94)=3.04, p=.052, \eta_{\mathrm{p}}{ }^{2}=.06$ (see Fig. 2).

IB increased significantly between the $200 \mathrm{~ms}$ and $250 \mathrm{~ms}$ delay, $t(47)=5.49, M_{\text {Diff(200 vs. } 250 \mathrm{~ms})}=22.95, S E_{\text {Diff(200 vs. } 250}$ $\mathrm{ms})=4.18, p<.001$, and between the $250 \mathrm{~ms}$ and $300 \mathrm{~ms}$ delay, $t(47)=3.26, M_{\text {Diff(250 vs. } 300 \mathrm{~ms})}=19.86, S E_{\text {Diff(250 vs. }}$ $300 \mathrm{~ms})=6.10, p=.002$.

IB for actions The data of two participants had to be excluded from the exploratory IB analysis for the action due to a misunderstanding of the instructions in some of the blocks (judgement of effect instead of action). For the remaining 46 participants (33 females; mean age $=26$ years, $S D=9.26$, range: $18-61$ years; 45 right-handed), separate $t$ - tests were conducted to assess whether IB was significant for each condition. All conditions showed significant IB, predictable, $200 \mathrm{~ms}: t(45)=$ 4.04, $M=25.22, S E=6.24, p<.001 ; 250 \mathrm{~ms}: t(45)=4.28, M$ $=28.13, S E=6.57, p<.001 ; 300 \mathrm{~ms}: t(45)=3.89, M=26.96$, $S E=6.92, p<.001 ;$ unpredictable, $200 \mathrm{~ms}: t(45)=5.99, M=$ 28.58, $S E=4.78, p<.001 ; 250 \mathrm{~ms}: t(45)=5.76, M=28.99$, $S E=5.04, p<.001 ; 300 \mathrm{~ms}: t(45)=5.94, M=32.73, S E=$ $5.51, p<.001$.

In a within-subjects $3 \times 2$ ANOVA (delay and predictability), neither the main effect of delay, $F(2,90)=0.45, p>.250$, $\eta_{\mathrm{p}}{ }^{2}=.01 ; 200 \mathrm{~ms}: M=26.90, S E=4.25 ; 250 \mathrm{~ms}: M=28.56$, $S E=4.56 ; 300 \mathrm{~ms}: M=29.84, S E=5.31$, nor the main effect of predictability, $F(1,45)=0.30, p>.250, \eta_{\mathrm{p}}^{2}<.01$; predictable: $M=26.77, S E=5.81$; unpredictable: $M=30.10, S E=$ 4.80 , nor the interaction of delay $\times$ predictability, $F(2,90)=$ $0.32, p>.250, \eta_{\mathrm{p}}{ }^{2}<.01$, were significant (see Fig. 3).

\section{Experiment 2}

In Experiment 2, we assessed IB for an extended range of effect delays and employed the delays of $100 \mathrm{~ms}, 250 \mathrm{~ms}$, and $400 \mathrm{~ms}$. IB for the effect delay of $100 \mathrm{~ms}$ is especially interesting because, in this condition, a strong IB for the effect might indicate a perceived reversal of action and effect (assumed that the temporal perception of actions is not biased in the same direction in this condition).

\section{Method}

Participants Forty-five participants (29 females; mean age $=26$ years, $S D=5.97$, range: $19-49$ years; 42 right-handed) were tested in exchange for 15 euros or course credit. The data of three further participants had to be discarded from the IB analysis of the effect due to a misunderstanding of the instructions in some of the blocks (judgment of action instead of effect). 


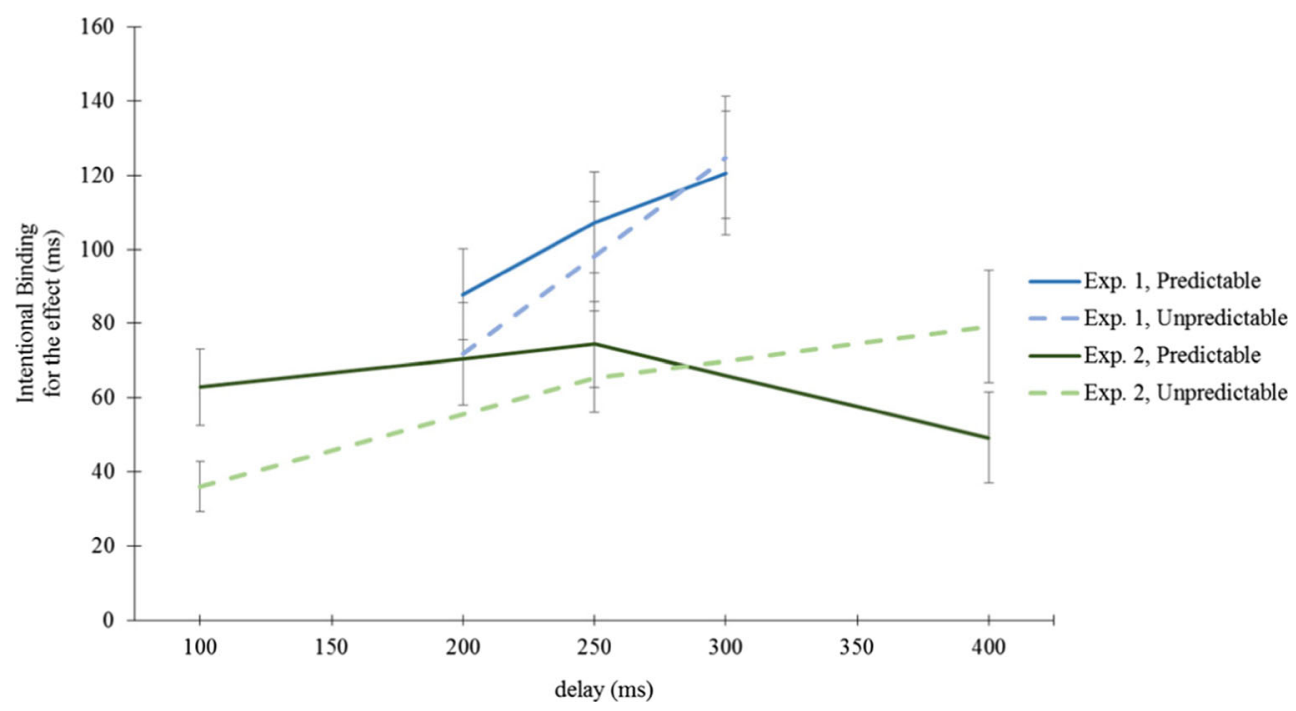

Fig. 2 Intentional binding for the effect depending on effect delay and delay predictability for Experiment 1, with delays $200 \mathrm{~ms}, 250 \mathrm{~ms}$, $300 \mathrm{~ms}$, and Experiment 2, with delays $100 \mathrm{~ms}, 250 \mathrm{~ms}, 400 \mathrm{~ms}$.

Intentional binding for the effects is depicted on the $y$-axis with positive values (see Method). Error bars represent standard errors (Color figure online)

Apparatus, stimuli, and procedure Apparatus, stimuli, and procedure were similar to Experiment 1, except that effect delays were $100 \mathrm{~ms}, 250 \mathrm{~ms}$, and $400 \mathrm{~ms} ; 2.23 \%$ of the trials were discarded because they differed more than $+/-2.5 \mathrm{~ms}$ from the participant's mean estimate in the respective condition.

\section{Results}

IB for effects To assess whether IB was significant for each condition, we conducted separate $t$ - tests. All conditions showed significant IB, predictable, $100 \mathrm{~ms}: t(44)=6.09, M$ $=62.90, S E=10.33, p<.001 ; 250 \mathrm{~ms}: t(44)=6.48, M=$ 74.37, $S E=11.47, p<.001 ; 400 \mathrm{~ms}: t(44)=4.03, M=49.24$, $S E=12.23, p<.001 ;$ unpredictable, $100 \mathrm{~ms}: t(44)=5.29, M=$
35.95, $S E=6.79, p<.001 ; 250 \mathrm{~ms}: t(44)=7.16, M=65.30$, $S E=9.12, p<.001 ; 400 \mathrm{~ms}: t(44)=5.20, M=79.23, S E=$ $15.24, p<.001$.

In a within-subjects $3 \times 2$ ANOVA (delay and predictability) neither delay, $F(2,88)=2.15, p=.123, \eta_{\mathrm{p}}{ }^{2}<.05 ; 100 \mathrm{~ms}: M=$ 49.43, $S E=7.11 ; 250 \mathrm{~ms}: M=69.84, S E=8.58 ; 400 \mathrm{~ms}: M=$ 64.23, $S E=12.76$, nor predictability were significant, $F(1,44)$ $=0.05, p>.250, \eta_{\mathrm{p}}^{2}<.01 ;$ predictable: $M=62.17, S E=8.75$; unpredictable: $M=60.16, S E=9.24$. The interaction delay $\times$ predictability was significant, $F(2,88)=14.78, p<.001, \eta_{\mathrm{p}}{ }^{2}$ $=.25$ (see Fig. 2).

The interaction was driven by a significant increase of IB between the $100 \mathrm{~ms}$ and $250 \mathrm{~ms}$ delay for unpredictably varying delays, $t(44)=4.04, M_{\text {Diff(100 vs. } 250 \mathrm{~ms})}=29.35, S E_{\text {Diff(100 }}$

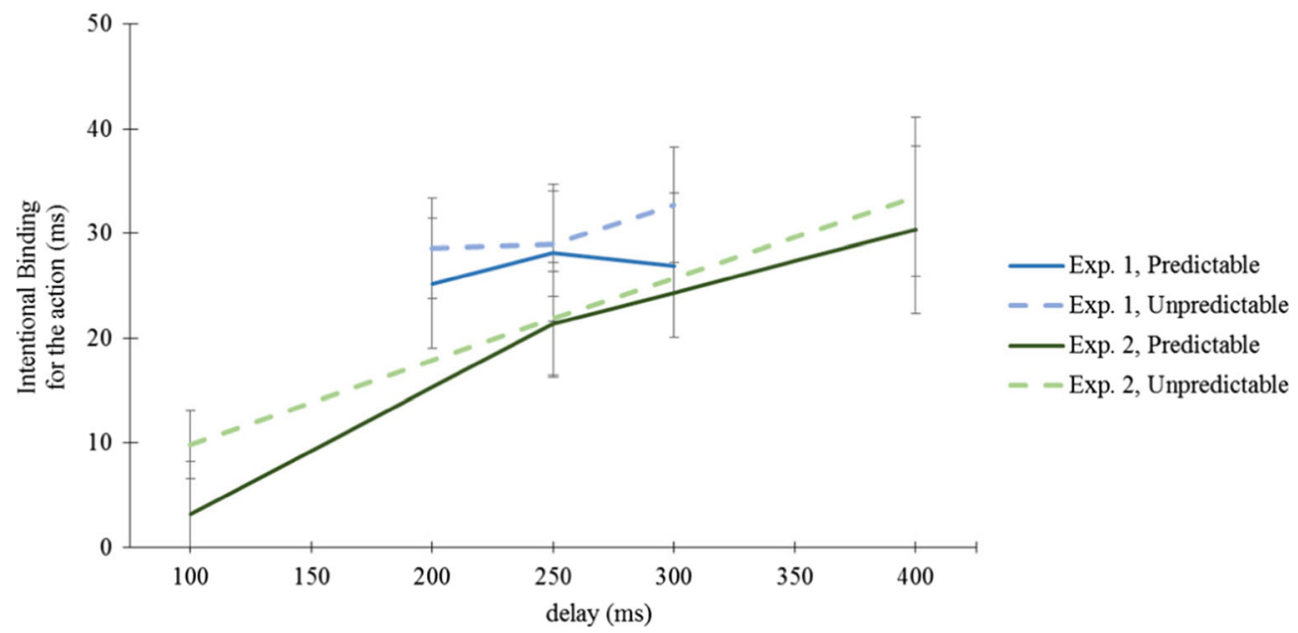

Fig. 3 Intentional binding for the action depending on delay and predictability for Experiment 1 (delays: $200 \mathrm{~ms}, 250 \mathrm{~ms}, 300 \mathrm{~ms}$ ) and Experiment 2 (delays: $100 \mathrm{~ms}, 250 \mathrm{~ms}, 400 \mathrm{~ms}$ ). Intentional binding for the actions is depicted on the $y$-axis, with positive values (see Method). Error bars represent standard errors (Color figure online) 
vs. $250 \mathrm{~ms})=7.27, p=.001$, and by a marginally significant decrease between the $250 \mathrm{~ms}$ and $400 \mathrm{~ms}$ delay for predictably varying delays, $t(44)=2.29, M_{\text {Diff }(250 \text { vs. } 400 \mathrm{~ms})}=-25.13$, $S E_{\text {Diff(250 vs. } 400 \mathrm{~ms})}=10.96, p=.08$. All other contrasts were not significant.

IB for actions Six of the initial 48 participants were excluded from the exploratory analysis due to a misunderstanding of the instructions in some of the blocks (judgment of effect instead of action; remaining 42 participants: 28 females; mean age $=$ 26 years, $S D=5.93$, range: $19-49$ years; 39 right-handed). To assess whether IB was significant for each condition, we conducted separate $t$ - tests. IB was insignificant for predictable, 100 ms: $t(41)=0.64, M=3.20, S E=4.97, p>.250$, and significant for predictable, $250 \mathrm{~ms}: t(41)=4.20, M=21.34$, $S E=5.08, p<.001 ; 400 \mathrm{~ms}: t(41)=3.79, M=30.35$, $S E=8.01, p<.001 ;$ unpredictable, $100 \mathrm{~ms}: t(41)=3.02$, $M=9.80, S E=3.24, p=.004 ; 250 \mathrm{~ms}: t(41)=4.05$, $M=21.87, S E=5.40, p<.001 ; 400 \mathrm{~ms}: t(41)=4.40$, $M=33.48, S E=7.62, p<.001$.

A within-subjects $3 \times 2$ ANOVA (delay and predictability) showed an increase of action IB with delay, $F(2,82)=15.58$, $p<.001, \eta_{\mathrm{p}}{ }^{2}=.28 ; 100 \mathrm{~ms}: M=6.50, S E=3.22 ; 250 \mathrm{~ms}$ : $M=21.61, S E=4.00 ; 400 \mathrm{~ms}: M=31.92, S E=6.21$. Neither the main effect of predictability, $F(1,41)=0.29, p>.250, \eta_{\mathrm{p}}{ }^{2}$ <.01; predictable: $M=18.30, S E=4.93$; unpredictable: $M=21.72, S E=5.00$, nor the interaction of delay $\times$ predictability, $F(2,82)=0.41, p>.250, \eta_{\mathrm{p}}{ }^{2}=.01$, were significant (see Fig. 3).

IB increased significantly between the $100 \mathrm{~ms}$ and $250 \mathrm{~ms}$ delay, $t(41)=4.26, M_{\text {Diff(100 vs. } 250 \mathrm{~ms})}=15.11, S E_{\text {Diff(100 vs. } 250}$ $\mathrm{ms})=3.54, p<.001$, and between the $250 \mathrm{~ms}$ and $400 \mathrm{~ms}$ delay, $t(41)=2.50, M_{\text {Diff(250 vs. } 400 \mathrm{~ms})}=10.31, S E_{\text {Diff(250 vs. }}$ $400 \mathrm{~ms})=4.13, p=.017$.

\section{Discussion}

An important and contentious issue in IB research is, whether and how IB is modulated by the temporal delay of the effect (e.g., Haggard et al., 2002; Humphreys \& Buehner, 2009; Nolden et al., 2012; Wen et al., 2015). We investigated the impact of effect delay and temporal predictability on IB magnitude around the ergonomically important delay of $250 \mathrm{~ms}$ (Seow, 2008; Smith \& Mosier, 1986). IB was measured as the temporal shift of the estimated point in time of the effect. When employing a small range $(200 \mathrm{~ms}, 250 \mathrm{~ms}, 300 \mathrm{~ms}$; Experiment 1) we found a monotonous increase of IB with effect delay. With a more extended range $(100 \mathrm{~ms}, 250 \mathrm{~ms}$, $400 \mathrm{~ms}$; Experiment 2) the increase turned to a decrease for $400 \mathrm{~ms}$, at least for temporally predictable delays. In other conditions, temporal predictability did not show any effect. These findings substantially extend our knowledge of the dynamics of IB because the only previous study scrutinizing the dynamics of IB in the sense of a shift of the perceived point in time of the effect found a monotonous decrease of IB in the range of $250 \mathrm{~ms}$ to $650 \mathrm{~ms}$ (Haggard et al., 2002). In the following, we discuss our findings in relation to the design of human-machine interfaces, to implications for a potential perceived reversal of the causal order for short delays, and to the well-researched dynamics of IB in the sense of a shortening of the perceived duration of action-effect intervals. However, beforehand, we summarize our exploratory findings concerning IB for the action.

In addition to the perceived temporal shift of the effect toward the action, we also analyzed the perceived shift of the action toward the effect. To our knowledge, the present study is the first one to scrutinize the influence of effect delay and temporal predictability on action IB. For the extended delay range (Experiment 2), we observed a monotonous increase of action IB with increasing delay. Thus, the duration of the delay following action execution influenced the perceived point in time of the action. Whereas some previous results primarily favor prospective processes to explain action IB (e.g., Haggard \& Clark, 2003), our results, suggest reafferent, that is, postdictive processes to impact on action IB (e.g., Yarrow \& Obhi, 2014). These findings are in line with recent studies by Moore and Haggard (2008) and Wolpe, Haggard, Siebner, and Rowe (2013), suggesting that action binding is influenced by external consequence, whereas tone binding is influenced by changes to the motor cortex (see also Moore, Ruge, Wenke, Rothwell, \& Haggard, 2010).

The finding that IB for the effect, as a measure of sense of agency, increases for short delays has important implications concerning human-machine interfaces. Much effort is taken to improve human-machine interfaces by introducing faster response times of machines (Seow, 2008). This is in line with previous studies showing sense of agency decreasing monotonously with delay (Wen et al., 2015). In contrast, our results showed an initial increase of IB for shorter delays. This, however, crucially relies on the assumption that IB is a reliable measure of sense of agency (Moore et al., 2009). Our results might give rise to calling this presumption into question. IB and sense of agency might dissociate for short delays. For short effect delays, and especially for effect delays of zero (e.g., immediate effects), it seems quite plausible to assume weak IB, because this prevents a violation of the order principle of causality between action and effect (see Introduction). Hence, using IB as an implicit measure of sense of agency might be restricted to longer delays.

However, when one assumes that IB does indeed reflect sense of agency, our results imply that shortening response latencies must be considered cautiously. If the effect follows the action instantaneously, sense of agency might be reduced. The effect might be attributed to external sources, instead of to the causing action, especially if users are not used to 


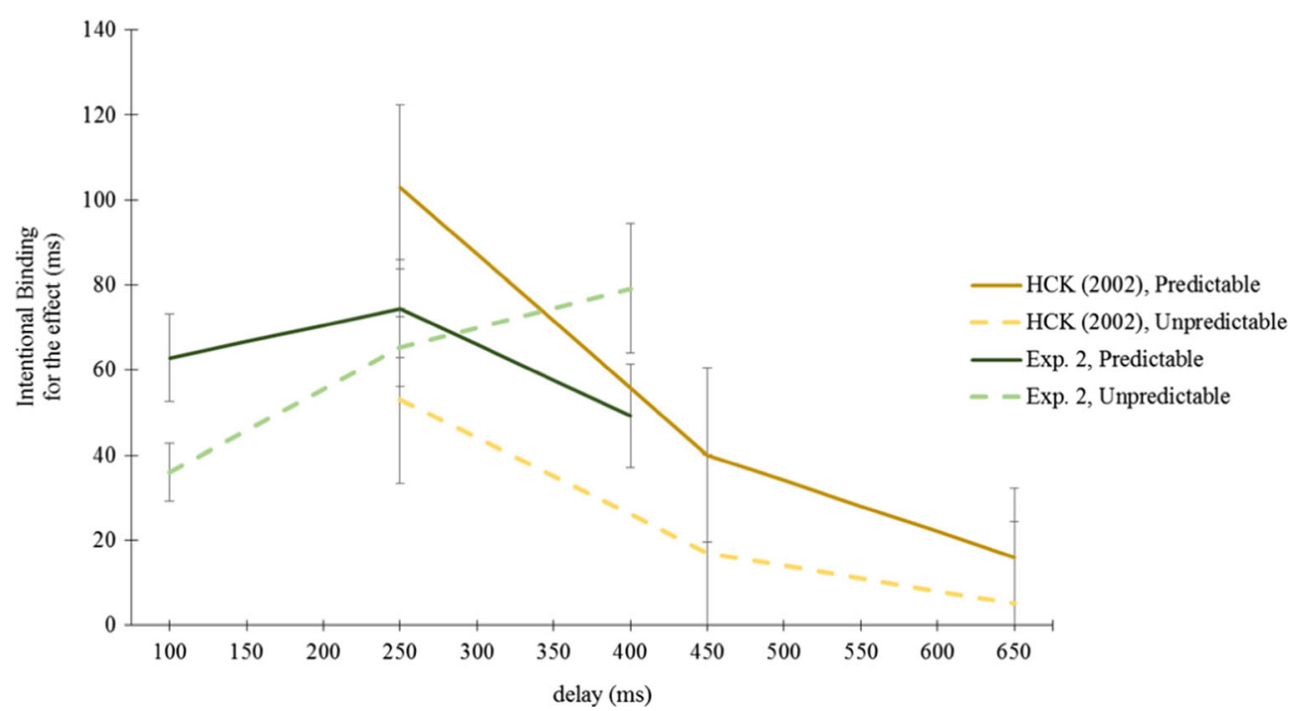

Fig. 4 Intentional binding for the effect depending on effect delay and delay predictability for the study by Haggard et al. (2002), abbreviated "HCK (2002)" with delays $250 \mathrm{~ms}, 450 \mathrm{~ms}, 650 \mathrm{~ms}$; and Experiment 2

with delays $100 \mathrm{~ms}, 250 \mathrm{~ms}, 400 \mathrm{~ms}$. Intentional binding for the effects is depicted on the $y$-axis with positive values (see Method). Error bars represent standard errors (Color figure online)

immediate machine responses. Previous results showed more sense of agency for effects occurring frequently after their specific delay (Haering \& Kiesel, 2015, 2016) as well as faster response times to these effects (Haering \& Kiesel, 2012).

Our results concerning lower IB for short delays might be due to the method employed. Probably, other measures for IB might lead to different conclusions. For example, simultaneity judgment tasks, where participants cause an effect and have to indicate whether it occurred simultaneously with or was succeeded by another action-unrelated stimulus. Although Wenke and Haggard (2009) compared simultaneity judgments only for two action unrelated stimuli, that is, two randomly occurring shocks that were presented between an action and its resulting effect, they showed lower discrimination for these two action unrelated stimuli if they occurred early after action execution (150 ms delay) in comparison to late after action execution ( $450 \mathrm{~ms}$ to $850 \mathrm{~ms}$ delay). Thus, simultaneous judgment tasks might result in sense of agency being higher for shorter delays. Therefore, it should be noted, that any conclusions from our results might be an artefact of the employed method.

With regard to IB for effects, our results complement results by Haggard et al. (2002). In their study, longer effect delays were applied, and IB decreased monotonously with increasing delays. Consequently, we conjecture an increase of IB for short delays up to a maximum at about $200 \mathrm{~ms}$ to $400 \mathrm{~ms}$, which turns into a monotonous decrease of IB for longer delays. Interestingly, the exact range of increase and decrease of IB seems to differ for predictable and unpredictable effect delays. In Experiment 2, we observed an interaction of effect delay and temporal predictability, indicating that IB declined between $250 \mathrm{~ms}$ and $400 \mathrm{~ms}$ effect delay for temporally predictable effects, while IB for unpredictable effects did not differ significantly for these effect delays. This finding is in line with the interaction between effect delay and temporal predictability found by Haggard et al. (2002). Their results suggest a faster decrease of IB for predictable in comparison to unpredictable effects (see Fig. 4).

Further investigation is necessary to specify more precisely at which effect delays IB increases and decreases under certain conditions. However, the convergent results speak for a dissociation in the increase and decrease of IB for predictable in comparison to unpredictable effects. One reason might be that the early occurring trials in the temporally unpredictable condition foster also the binding in trials where the effect occurs later. On the other hand, in the temporally predictable condition with late delays, there are no such early occurring stimuli that might further bias the effect to be perceived earlier. This might lead to the faster decrease of IB for predictable in comparison to unpredictable effects with delay duration. However, this potential explanation for differences in IB between predictable and unpredictable effects are very speculative and require further investigation. Nevertheless, together with Haggard et al.'s (2002) results, it might be concluded, cautiously, that the exact characteristics and context of action and effect might determine at which effect delay IB increases and decreases.

Some literature has used duration estimates to investigate how effect delay influences IB (e.g., Humphreys \& Buehner, 2009; Nolden et al., 2012; Wen et al., 2015). Yet for clarifying whether a shift of points in time of action and effect occurred or not, these methods lack differentiation. According to common accounts of human time perception, IB might be caused by a slowing of an internal clock, or by a shift of perceived points in time of action and effect (e.g., Wenke \& Haggard, 2009). Of course, both mechanisms are not mutually exclusive 
and might both contribute to IB. In duration estimation tasks, IB might either be caused by a slowing of the internal clock or by a shift of perceived points in time of action and effect. Thus, it remains unclear which of these mechanisms occurred in the duration estimation tasks. If IB is measured with the clock paradigm, IB can unambiguously be attributed to shifts in perceived points in time. Consequently, our results offer differential insight on the distinct mechanisms that underlie the impact of effect delay and temporal predictability on IB.

However, Haggard et al.'s (2002) results that show a monotonously decreasing relationship of effect delay and IB differ from results conducted by duration estimation tasks, as studies with the latter paradigm found IB to increase up to delays of $4 \mathrm{~s}$ (Humphreys \& Buehner, 2009). This contrast suggests that both potential aspects of IB - a slowing of the internal clock and a shift of the perceived points in time of action and effect-are differentially affected by delay duration. Therefore, probably, the contrary results might be due to the studies using different methods that might be sensitive to different mechanisms contributing to IB. These mechanisms might be influenced in different ways by longer delays, thereby leading to the contrary results found. Further investigations are necessary for clarifying the distinct impacts of underlying mechanisms on IB as well as their interplay.

Overall, our results clearly extend previous findings concerning the relation of effect delay and IB. The only previous study investigating the influence of delay on the perceived temporal shift of the effect found a monotonously decreasing relationship (Haggard et al., 2002). Yet, combining their and our results, it might suggest an inverted U-shaped relation of effect delay and IB. For effect delays shorter than $200 \mathrm{~ms}$ to $400 \mathrm{~ms}$ IB seems to increase, while it decreases for longer ones (Haggard et al., 2002). However, this pattern follows only indirectly from an integrative interpretation of our and Haggard et al.'s (2002) study, and needs to be confirmed by future research.

Author note Miriam Ruess, Roland Thomaschke, and Andrea Kiesel, Cognition, Action, and Sustainability Unit, Department of Psychology, Albert-Ludwigs-University of Freiburg.

The study was funded by Deutsche Forschungsgemeinschaft grant No. KI.1388/3-2.

We thank S. K. Poonian and an anonymous reviewer for very constructive comments on a previous version of this paper.

Raw data are available at https://osf.io/s6z3y/\#

\section{References}

Berberian, B., Sarrazin, J.-C., LeBlaye, P., \& Haggard, P. (2012). Automation technology and sense of control: A window on human agency. PloS One, 7(3), e34075. doi:10.1371/journal.pone.0034075

Desantis, A., Hughes, G., \& Waszak, F. (2012). Intentional binding is driven by the mere presence of an action and not by motor prediction. PloS One, 7(1), e29557. doi:10.1371/journal.pone.0029557
Engbert, K., Wohlschläger, A., \& Haggard, P. (2008). Who is causing what? The sense of agency is relational and efferent-triggered. Cognition, 107(2), 693-704. doi:10.1016/j.cognition.2007.07.021

Haering, C., \& Kiesel, A. (2012). Time in action contexts: Learning when an action effect occurs. Psychological Research, 76(3), 336-344. doi:10.1007/s00426-011-0341-8

Haering, C., \& Kiesel, A. (2014). Intentional binding is independent of the validity of the action effect's identity. Acta Psychologica, 152, 109-119. doi:10.1016/j.actpsy.2014.07.015

Haering, C., \& Kiesel, A. (2015). Was it me when it happened too early? Experience of delayed effects shapes sense of agency. Cognition, 136, 38-42. doi:10.1016/j.cognition.2014.11.012

Haering, C., \& Kiesel, A. (2016). Time perception and the experience of agency. Psychological Research, 80(2), 286-297. doi:10.1007/ s00426-015-0654-0

Haggard, P. (2005). Conscious intention and motor cognition. Trends in Cognitive Sciences, 9(6), 290-295. doi:10.1037/a0028566

Haggard, P., \& Clark, S. (2003). Intentional action: Conscious experience and neural prediction. Consciousness and Cognition, 12(4), 695707. doi:10.1016/S1053-8100(03)00052-7

Haggard, P., Clark, S., \& Kalogeras, J. (2002). Voluntary action and conscious awareness. Nature Neuroscience, 5(4), 382-385. doi:10. $1038 / \mathrm{nn} 827$

Haggard, P., Poonian, S. K., \& Walsh, E. (2009). Representing the consequences of intentionally inhibited actions. Brain Research, 1286, 106-113. doi:10.1016/j.brainres.2009.06.020

Hume, D. (1874). A treatise of human nature. In T. H. Green \& T. H. Grose (Eds.), The philosophical works of David Hume. London, UK: Longmans, Green.

Humphreys, G. R., \& Buehner, M. J. (2009). Magnitude estimation reveals temporal binding at super-second intervals. Journal of Experimental Psychology: Human Perception and Performance, 35(5), 1542-1549. doi:10.1037/a0014492

Libet, B., Gleason, C. A., Wright, E. W., \& Pearl, D. K. (1983). Time of conscious intention to act in relation to onset of cerebral activity (readiness potential): The unconscious initiation of a freely voluntary act. Brain, 106(3), 623-642. doi:10.1093/brain/106.3.623

Limerick, H., Coyle, D., \& Moore, J. W. (2014). The experience of agency in human-computer interactions: A review. Frontiers in Human Neuroscience, 8(643), 87-96. doi:10.3389/fnhum.2014. 00643

Moore, J. W., \& Haggard, P. (2008). Awareness of action: Inference and prediction. Consciousness and Cognition, 17(1), 136-144. doi:10. 1016/j.concog.2006.12.004

Moore, J. W., \& Haggard, P. (2010). Intentional binding and higher order agency experience. Consciousness and Cognition, 19(1), 490-491. doi:10.1016/j.concog.2009.11.007

Moore, J. W., Ruge, D., Wenke, D., Rothwell, J. C., \& Haggard, P. (2010). Disrupting the experience of control in the human brain: Pre-supplementary motor area contributes to the sense of agency. Proceedings of the Royal Society of London B: Biological Sciences, 277(1693), 2503-2509. doi:10.1098/rspb.2010.0404

Moore, J. W., Wegner, D. M., \& Haggard, P. (2009). Modulating the sense of agency with external cues. Consciousness and Cognition, 18(4), 1056-1064. doi:10.1016/j.concog.2009.05.004

Mudd, S. A. (1963). Spatial stereotypes of four dimensions of pure tone. Journal of Experimental Psychology, 66(4), 347-352. doi:10.1037/ h0040045

Nolden, S., Haering, C., \& Kiesel, A. (2012). Assessing intentional binding with the method of constant stimuli. Consciousness and Cognition, 21(3), 1176-1185. doi:10.1016/j.concog.2012.05.003

Poonian, S. K., \& Cunnington, R. (2013). Intentional binding in selfmade and observed actions. Experimental Brain Research, 229(3), 419-427. doi:10.1007/s00221-013-3505-5

Schneider, W., Eschmann, A., \& Zuccolotto, A. (2012). E-Prime user's guide. Pittsburgh, PA: Psychology Software Tools. 
Seow, S. C. (2008). Designing and engineering time: The psychology of time perception in software. Salt Lake City, UT: Addison-Wesley Professional.

Smith, S. L., \& Mosier, J. N. (1986). Guidelines for designing user interface software. Bedford, MA: Mitre Corporation.

Stenner, M.-P., Bauer, M., Machts, J., Heinze, H.-J., Haggard, P., \& Dolan, R. J. (2014). Re-construction of action awareness depends on an internal model of action-outcome timing. Consciousness and Cognition, 25(1), 11-16. doi:10.1016/j. concog.2014.01.007

Wen, W., Yamashita, A., \& Asama, H. (2015). The influence of actionoutcome delay and arousal on sense of agency and the intentional binding effect. Consciousness and Cognition, 36, 87-95. doi:10. 1016/j.concog.2015.06.004
Wenke, D., \& Haggard, P. (2009). How voluntary actions modulate time perception. Experimental Brain Research, 196(3), 311-318. doi:10. 1007/s00221-009-1848-8

Wolpe, N., Haggard, P., Siebner, H. R., \& Rowe, J. B. (2013). Cue integration and the perception of action in intentional binding. Experimental Brain Research, 229(3), 467-474. doi:10.1007/ s00221-013-3419-2

Wundt, W. (1887). Grundzüge der physiologischen Psychologie [Principles of physiological psychology] (3rd ed.). Leipzig, Germany: Wilhelm Engelman.

Yarrow, K., \& Obhi, S. S. (2014). Temporal perception in the context of action. In V. Arstila \& D. Lloyd (Eds.), Subjective time: The philosophy, psychology, and neuroscience of temporality (pp. 455-476). Cambridge, MA: MIT Press. 